\title{
On the Error in the Numerical Integration of Chebyshev Polynomials
}

\author{
By D. Nicholson, P. Rabinowitz, N. Richter and D. Zeilberger
}

\begin{abstract}
A general method is described to compute the exact error in the numerical integration of a given polynomial by certain types of integration rules. This method is applied to get exact errors in the integration of certain Chebyshev polynomials of the first kind by Gauss and Lobatto rule and asymptotic errors in the integration of Chebyshev polynomials of both kinds by Gauss, Lobatto and Radau rules.
\end{abstract}

1. Introduction. In this work, we consider the error in the numerical integration of the Chebyshev polynomials of the first and second kind, $T_{n}(x), U_{n}(x)$ by Gauss, Lobatto and Radau integration rules. We first derive explicit expressions for the error in the numerical integration of $T_{2 n+2 k}(x)$ by an $n$-point Gaussian integration rule and by an $(n+1)$-point Lobatto rule, for $k=0,1,2$. The method used for deriving these expressions can be applied for any $k \geqq 0$, although we only go up to $k=2$ since the number of algebraic manipulations grows rapidly with $k$. It can also be applied to $U_{2 n+2 k}(x)$ and in fact to any sequence of polynomials and in addition can be applied in connection with any numerical integration rule. We then derive asymptotic expressions as $n$ tends to infinity for the error in the numerical integration of $T_{2 n+k}(x)$ and $U_{2 n+k}(x)$ for fixed $k \geqq 0$, using the three integration rules mentioned above. Actually, for the Gauss and Lobatto rules which are symmetric rules, the error in the numerical integration of Chebyshev polynomials of odd degree is identically zero so that we need consider only polynomials of even degree, $T_{2 n+2 k}(x)$ and $U_{2 n+2 k}(x)$.

2. The Error in Gauss and Lobatto Integration Using a Fixed Number of Points. The Gaussian rule of degree $2 n-1$ with respect to the interval $[-1,1]$ has the $n$ roots of the Jacobi polynomial $P_{n}^{(0,0)}(x)\left(=P_{n}(x)\right.$, the Legendre polynomial) as its $n$ abscissas [1, p. 33]. Denoting the error committed by applying this rule to $T_{k}(x)$ by $E_{n}\left(T_{k}\right)$, we have the following relations:

$$
\begin{aligned}
E_{n}\left(T_{k}\right) & =0, & & k \leqq 2 n-1, \\
E_{n}\left(T_{2 m+1}\right) & =0, & & m \geqq 0 .
\end{aligned}
$$

The Lobatto rule of degree $2 n-1$ with respect to the interval $[-1,1]$ consists of $n+1$ abscissas, of which $n-1$ are the roots of the Jacobi polynomial $P_{n-1}^{(1,1)}(x)$, and the other two are the endpoints of the interval [1, p. 37]. Denoting the error

Received April 16, 1970, revised June 25, 1970.

AMS 1969 subject classifications. Primary 6555, 6580; Secondary 4144.

Key words and phrases. Numerical integration, exact error, asymptotic error, Jacobi polynomial, Chebyshev polynomial, Gauss rule, Lobatto rule, Radau rule. 
committed by applying this rule to $T_{k}(x)$ by $E_{n+1}\left(T_{k}\right)$, we have the following similar relations:

$$
\begin{aligned}
E_{n+1}\left(T_{k}\right) & =0, & & k \leqq 2 n-1, \\
E_{n+1}\left(T_{2 m+1}\right) & =0, & & m \geqq 0 .
\end{aligned}
$$

In the Gaussian case let us now write

$$
T_{2 n+2 k}(x)=\left[P_{n}^{(0,0)}(x)\right]^{2} \sum_{i=0}^{k} a_{i}^{(k)} x^{2 i}+Q_{2 n-2}(x),
$$

where $Q_{2 n-2}(x)$ is the remainder in the division of $T_{2 n+2 k}(x)$ by $\left[P_{n}^{(0,0)}(x)\right]^{2}$. We then have the following expression for $E_{n}\left(T_{2 n+2 k}\right)$ :

$$
\begin{aligned}
E_{n}\left(T_{2 n+2 k}\right) & =\int_{-1}^{1}\left[P_{n}^{(0,0)}(x)\right]^{2} \sum_{i=0}^{k} a_{i}^{(k)} x^{2 i} d x \\
& =\sum_{i=0}^{k} a_{i}^{(k)} \int_{-1}^{1}\left[x^{i} P_{n}^{(0,0)}(x)\right]^{2} d x .
\end{aligned}
$$

This is true since $E_{n}\left(Q_{2 n-2}\right)=0$ and since $\left[P_{n}^{(0,0)}(x)\right]^{2}$ vanishes at the abscissas of the integration rule. The integrals in (2) can be evaluated explicitly as follows: By repeated use of the following recurrence formula [2, p. 83]

$$
(2 n+1) x P_{n}^{(0,0)}(x)=(n+1) P_{n+1}^{(0,0)}(x)+n P_{n-1}^{(0,0)}(x),
$$

we can replace $x^{i} P_{n}^{(0,0)}(x)$ by $\sum_{i=-i}^{i} c_{j}^{(i)} P_{n+i}^{(0,0)}(x)$. By the orthogonality of the $P_{n}^{(0,0)}(x)$, we have that

$$
\int_{-1}^{1}\left[\sum_{i=-i}^{i} c_{i}^{(i)} P_{n+i}^{(0,0)}(x)\right]^{2} d x=\sum_{j=-i}^{i} c_{i}^{(i) 2} \int_{-1}^{1}\left[P_{n+j}^{(0,0)}(x)\right]^{2} d x
$$

and hence, using the formula [2, p. 82]:

$$
\int_{-1}^{1}\left[P_{n}^{(0,0)}(x)\right]^{2} d x=\frac{2}{2 n+1}
$$

we have finally that:

$$
\begin{aligned}
E_{n}\left(T_{2 n+2 k}\right) & =\sum_{i=0}^{k} a_{i}^{(k)} \sum_{i=-i}^{i} c_{i}^{(i) 2} \int_{-1}^{1}\left[P_{n+j}^{(0,0)}(x)\right]^{2} d x \\
& =2 \sum_{i=0}^{k} a_{i}^{(k)} \sum_{i=-i}^{i} c_{i}^{(i) 2} /(2 n+2 j+1) .
\end{aligned}
$$

For the Lobatto case, we apply the same method, replacing $\left[P_{*}^{(0,0)}(x)\right]^{2}$ by $\left(1-x^{2}\right)\left[P_{n-1}^{(1,1)}(x)\right]^{2}$ in (1). In this case, we use the corresponding recurrence formula $[2$, p. 83]:

$$
(2 n+3) x P_{n}^{(1,1)}(x)=(n+1) P_{n}^{(1,1)}(x)+(n+2) P_{n-1}^{(1,1)}(x)
$$

and the following formula for the evaluation of the integrals:

$$
\int_{-1}^{1}\left(1-x^{2}\right)\left[P_{n}^{(1,1)}(x)\right]^{2} d x=2(n+2)(n+1) / 2 n+3 .
$$


TABLE I

Exact Error in the Integration of $T_{2 n+2 k}, k=0,1,2$, by $n$-Point Gauss and $(n+1)$-Point Lobatto Rules

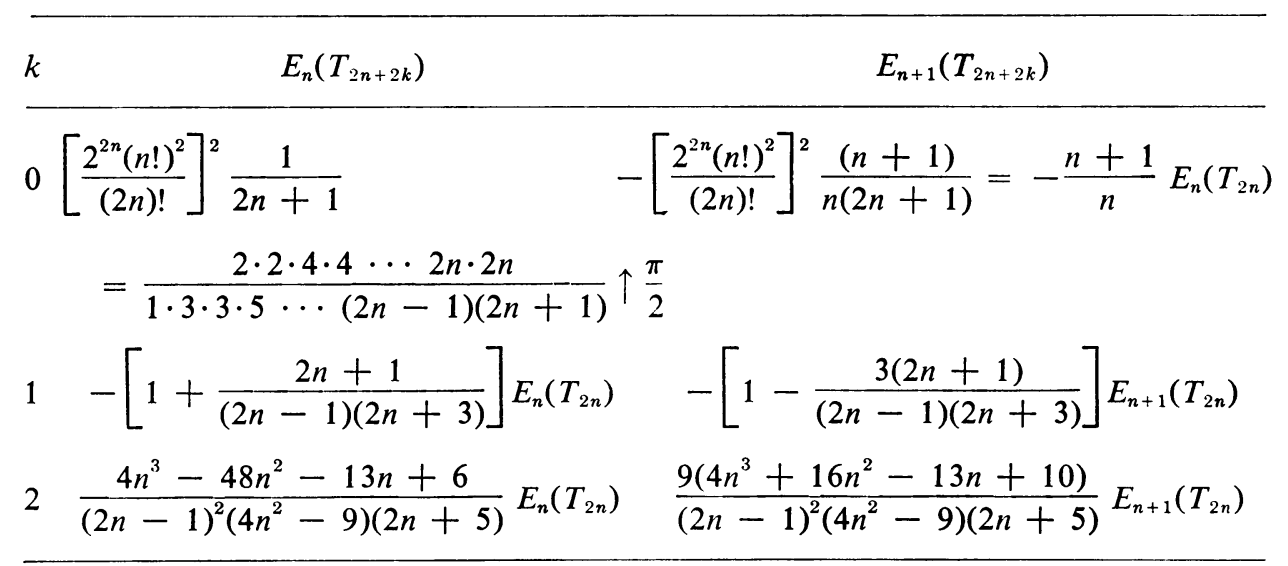

The results in the Gauss and Lobatto cases for $k=0,1,2$ are given in Table I. (For $k=0$, the results can also be obtained from the standard error terms since in this case $f^{(2 n)}(\xi)$ is a constant.)

Remark. This method is applicable to the calculation of the error in the numerical integration of any polynomial $P_{n+k}(x)$ by any integration rule of degree $n-1$ with respect to an arbitrary weight function $w(x)$. We just replace (1) by the following:

$$
P_{n+k}(x)=\Pi_{n}(x) \sum_{i=0}^{n} a_{i}^{(k)} x^{i}+Q_{n-1}(x), \quad k \geqq 0,
$$

where $\Pi_{n}(x)=r(x)[s(x)]^{2}$ with $r(x)=\prod_{i=1}^{n_{1}}\left(x-x_{i}\right), s(x)=\prod_{i=1}^{n_{2}}\left(x-y_{i}\right)$ and $n_{1}+2 n_{2}=n$. The points $x_{1}, \cdots, x_{n_{1}}, y_{1}, \cdots, y_{n_{2}}$ are the abscissas of the integration rule with $s(x)$ orthogonal to all polynomials of degree $\leqq n_{2}-1$, with respect to the weight function $w(x) r(x)$. The case $n_{1}=0$ corresponds to integration rules of Gaussian type while $n_{2}=0$ corresponds to interpolatory rules.

3. Asymptotic Expressions for the Error in Gauss and Lobatto Rules as $n \rightarrow \infty$. To get the asymptotic behavior as $n \rightarrow \infty$ of the error in the numerical integration of $T_{2 n+2 k}(x)$ and $U_{2 n+2 k}(x)$ where $k$ is held fixed, we use the method of the previous section except that we replace $P_{n}^{(\alpha, \alpha)}(x)$ by its asymptotic formula due to Darboux [2, p. 194]:

$$
P_{n}^{(\alpha, \alpha)}(\cos \theta)=n^{-1 / 2} k(\theta) \cos (N \theta+\phi)+O\left(n^{-3 / 2}\right),
$$

where $k(\theta)=\pi^{-1 / 2}(\sin \theta / 2)^{-\alpha-1 / 2}, N=n+\alpha+\frac{1}{2}, \phi=-\left(\alpha+\frac{1}{2}\right) \pi / 2$, and $0<$ $\theta<\pi$.

Since we are interested in the case where $\alpha$ is an integer, we derive the following formula from (3):

$$
\begin{aligned}
{\left[P_{n}^{(\alpha, \alpha)}(x)\right]^{2} } & =\frac{1}{2 n} k^{2}(\theta)[1+\cos (2 N \theta+2 \phi)]+O\left(n^{-2}\right) \\
& =2^{2 \alpha}\left[1+(-1)^{\alpha} \sin (2 n+2 \alpha+1) \theta\right]\left[\pi n(\sin \theta)^{2 \alpha+1}\right]^{-1}+O\left(n^{-2}\right)
\end{aligned}
$$


If we now define $S_{n}^{\alpha}(x)=\left(1-x^{2}\right)^{\alpha}\left[P_{n-\alpha}^{(\alpha, \alpha)}(x)\right]^{2}$, we have that

$$
S_{n}^{\alpha}(\cos \theta)=2^{2 \alpha}\left[1+(-1)^{\alpha} \sin (2 n+1) \theta\right][\pi(n-\alpha) \sin \theta]^{-1}+O\left(n^{-2}\right) .
$$

Rewriting (1) with $x=\cos \theta$ and with $\left[P_{n}^{(0,0)}(x)\right]^{2}$ replaced by $S_{n}^{\alpha}(x)$, we get the following asymptotic relation for $T_{2 n+2 k}(x)$ :

$$
\begin{aligned}
T_{2 n+2 k}(\cos \theta)= & \cos (2 n+2 k) \theta \\
= & 2^{2 \alpha}\left[1+(-1)^{\alpha} \sin (2 n+1) \theta+O\left(n^{-1}\right)\right][(n-\alpha) \pi \sin \theta]^{-1} \\
& \cdot \sum_{i=0}^{k} b_{\alpha i}^{(k)} \cos 2 j \theta+Q_{2 n-2}^{\alpha}(\cos \theta),
\end{aligned}
$$

where

$$
\sum_{j=0}^{k} b_{\alpha i}^{(k)} \cos 2 j \theta=\sum_{i=0}^{k} a_{\alpha i}^{(k)}(\cos \theta)^{2 j}
$$

For $U_{2 n+2 k}(x)$, we get a similar relation:

$$
\begin{aligned}
U_{2 n+2 k}(\cos \theta)= & \sin (2 n+2 k+1) \theta / \sin \theta \\
= & 2^{2 \alpha}\left[1+(-1)^{\alpha} \sin (2 n+1) \theta+O\left(n^{-1}\right)\right][(n-\alpha) \pi \sin \theta]^{-1} \\
& \cdot \sum_{i=0}^{k} c_{\alpha i}^{(k)} \cos 2 j \theta+R_{2 n-2}^{\alpha}(\cos \theta) .
\end{aligned}
$$

Multiplying (4) and (5) respectively by $\sin \theta$, we get the following formulas:

$$
\begin{aligned}
\sin (2 n+ & 2 k+1) \theta-\sin (2 n+2 k-1) \theta \\
= & \frac{2^{2 \alpha+1}}{n \pi} \sum_{j=0}^{k} b_{\alpha i}^{(k)}\left[\cos 2 j \theta+O\left(n^{-1}\right)\right] \\
& +\frac{(-1)^{\alpha} 2^{2 \alpha}}{n \pi} \sum_{j=0}^{k} b_{\alpha i}^{(k)}\left[\sin (2 n+2 j+1) \theta+\sin (2 n-2 j+1) \theta^{\top}\right. \\
& +\tilde{Q}_{2 n-1}^{\alpha}(\sin \theta), \\
\sin (2 n+2 k & +1) \theta=\frac{2^{2 \alpha}}{n \pi} \sum_{j=0}^{k} c_{\alpha i}^{(k)}\left[\cos 2 j \theta+O\left(n^{-1}\right)\right] \\
& +\frac{(-1)^{\alpha} 2^{2 \alpha-1}}{n \pi} \sum_{j=0}^{k} c_{\alpha i}^{(k)}[\sin (2 n+2 j+1) \theta+\sin (2 n-2 j+1) \theta] \\
& +\tilde{R}_{2 n-1}^{(\alpha)}(\sin \theta),
\end{aligned}
$$

where $\widetilde{Q}_{2 n-1}^{\alpha}(\sin \theta)$ and $\tilde{R}_{2 n-1}^{\alpha}(\sin \theta)$ are odd polynomials of degree $2 n-1$ in $\sin \theta$ and are equal to certain linear combinations of $\sin (2 l+1) \theta, l=0, \cdots, n-1$.

Multiplying $\left(4^{\prime}\right)$ and $\left(5^{\prime}\right)$ respectively by $\sin (2 n+2 l+1) \theta, l=0, \cdots, k$, and integrating both sides between 0 and $\pi$, we get:

$$
\begin{aligned}
{\left[\delta_{k l}-\delta_{(k-1) l}\right] \frac{\pi}{2}=} & \frac{2^{2 \alpha+1}}{n \pi} \sum_{j=0}^{k} b_{\alpha i}^{(k)}\left[\frac{4 n+4 l+2}{(2 n+2 l+1)^{2}-4 j^{2}}+O\left(n^{-1}\right)\right] \\
& +\frac{(-1)^{\alpha} 2^{2 \alpha-1}}{n}\left[b_{\alpha i}^{(k)}+b_{\alpha 0}^{(k)} \delta_{0 l}\right], \quad l=0, \cdots, k,
\end{aligned}
$$


(7)

$$
\begin{aligned}
\delta_{k l} \frac{\pi}{2}= & \frac{2^{2 \alpha}}{n \pi} \sum_{i=0}^{k} c_{\alpha i}^{(k)}\left[\frac{4 n+4 l+2}{(2 n+2 l+1)^{2}-4 j^{2}}+O\left(n^{-1}\right)\right] \\
& +\frac{(-1)^{\alpha} 2^{2 \alpha-2}}{n}\left[c_{\alpha l}^{(k)}+c_{\alpha 0}^{(k)} \delta_{0 l}\right], \quad l=0, \cdots, k,
\end{aligned}
$$

where

$$
\begin{aligned}
\delta_{i k} & =0, & i \neq k, \\
& =1, & i=k .
\end{aligned}
$$

Here we have used the following well-known results:

$$
\text { (8) } \begin{aligned}
\int_{0}^{\pi} \sin k \theta \sin l \theta d \theta & =\frac{\pi}{2} \delta_{k l}, \quad k \geqq 1, \\
\int_{0}^{\pi} \sin k \theta \cos l \theta d \theta & =0, \quad k=l \text { or } k=0, \\
& =\frac{k}{k^{2}+l^{2}}\left[1+(-1)^{k+l+1}\right], \quad k \geqq 1, \quad k \neq l .
\end{aligned}
$$

The asymptotic solutions of the linear systems of Eqs. (6) and (7) are as follows:

$$
\begin{aligned}
& b_{\alpha l}^{(k)}=(-1)^{\alpha} n 2^{-2 \alpha} \pi\left[\delta_{k l}-\delta_{(k-1) l}\right]+O(1), \quad k \geqq 2, \quad l=0, \cdots, k, \\
& b_{\alpha 0}^{(1)}=(-1)^{\alpha+1} n 2^{-2 \alpha-1} \pi+O(1), \\
& b_{\alpha 1}^{(1)}=(-1)^{\alpha} n 2^{-2 \alpha} \pi+O(1), \\
& b_{\alpha 0}^{(0)}=(-1)^{\alpha} n 2^{-2 \alpha-1} \pi+O(1), \\
& c_{\alpha l}^{(k)}=(-1)^{\alpha} n 2^{-2 \alpha+1} \pi \delta_{k l}+O(1), \quad k \geqq 1, \quad l=0, \cdots, k, \\
& c_{\alpha 0}^{(0)}=(-1)^{\alpha} n 2^{-2 \alpha} \pi+O(1) .
\end{aligned}
$$

Returning now to the general form of (2), we get:

$$
\begin{aligned}
E\left(T_{2 n+2 k}\right) & =\int_{-1}^{1} S_{n}^{\alpha}(x)\left(\sum_{i=0}^{k} a_{\alpha i}^{(k)} x^{2 i}\right) d x \\
& =\int_{0}^{\pi} S_{n}^{\alpha}(\cos \theta)\left(\sum_{j=0}^{k} b_{\alpha i}^{(k)} \cos 2 j \theta\right) \sin \theta d \theta
\end{aligned}
$$

and a similar expression for $E\left(U_{2 n+2 k}\right)$ in which $b_{\alpha i}^{(k)}$ is replaced by $c_{\alpha i}^{(k)}$. Inserting the asymptotic expressions for $S_{n}^{\alpha}(\cos \theta), b_{\alpha i}^{(k)}$ and $c_{\alpha i}^{(k)}$ derived above, we get the following expressions:

$$
\begin{aligned}
E_{n+\alpha}\left(T_{2 n+2 k}\right)= & \int_{0}^{\pi}\left[(-1)^{\alpha}+\sin (2 n+1) \theta\right][\cos 2 k \theta-\cos (2 k-2) \theta] d \theta \\
& +O\left(n^{-1}\right), \quad k \geqq 2, \\
E_{n+\alpha}\left(T_{2 n+2}\right)= & \int_{0}^{\pi}\left[(-1)^{\alpha}+\sin (2 n+1) \theta\right]\left[\cos 2 \theta-\frac{1}{2}\right] d \theta+O\left(n^{-1}\right), \\
E_{n+\alpha}\left(T_{2 n}\right)= & \frac{1}{2} \int_{0}^{\pi}\left[(-1)^{\alpha}+\sin (2 n-1) \theta\right] d \theta+O\left(n^{-1}\right),
\end{aligned}
$$




$$
\begin{aligned}
E_{n+\alpha}\left(U_{2 n+2 k}\right) & =\int_{0}^{\pi}\left[(-1)^{\alpha}+\sin (2 n+1) \theta\right] 2 \cos 2 k \theta d \theta+O\left(n^{-1}\right), \quad k \geqq 1, \\
E_{n+\alpha}\left(U_{2 n}\right) & =\int_{0}^{\pi}\left[(-1)^{\alpha}+\sin (2 n+1) \theta\right] d \theta+O\left(n^{-1}\right) .
\end{aligned}
$$

Here the notation $E_{n+\alpha}$ refers to the error committed when using a rule whose general structure we shall describe in the forthcoming remark. If we now evaluate the above integrals using the formulas in (8), we get the final results:

$$
\begin{aligned}
E_{n+\alpha}\left(T_{2 n+2 k}\right) & =O\left(n^{-1}\right), \quad k \geqq 2, \\
E_{n+\alpha}\left(T_{2 n+2}\right) & =(-1)^{\alpha+1} \pi / 2+O\left(n^{-1}\right), \\
E_{n+\alpha}\left(T_{2 n}\right) & =(-1)^{\alpha} \pi / 2+O\left(n^{-1}\right), \\
E_{n+\alpha}\left(U_{2 n+2 k}\right) & =O\left(n^{-1}\right), \quad k \geqq 1, \\
E_{n+\alpha}\left(U_{2 n}\right) & =(-1)^{\alpha} \pi+O\left(n^{-1}\right) .
\end{aligned}
$$

Remark. Results (11) are valid for every nonnegative integer $\alpha$ and correspond to integration rules of degree $2 n-1$. For $\alpha=0,1$, the corresponding rules are the Gauss and Lobatto rules respectively while for $\alpha \geqq 2$ the rules are those which incorporate values of the integrand and its first $\alpha-1$ derivatives at the endpoints \pm 1 , namely:

$$
\int_{-1}^{1} f(x) d x \simeq \sum_{k=0}^{a-1} W_{k}\left[f^{(k)}(-1)+f^{(k)}(+1)\right]+\sum_{j=1}^{n-\alpha} A_{i} f\left(x_{i}\right)
$$

where $x_{1}, \cdots, x_{n-\alpha}$ are the roots of the Jacobi polynomial $P_{n-\alpha}^{(\alpha, \alpha)}(x)$.

The results for $a=0,1$ are included in Table II.

4. Asymptotic Expressions for the Error in Radau Rules as $n \rightarrow \infty$. The Radau rule of degree $2 n$ with respect to the interval $[-1,1]$ consists of $n+1$ abscissas, of which $n$ are the roots of the Jacobi polynomial $P_{n}^{(1,0)}(x)$ and the additional abscissa is at the point $x_{n+1}=1[1, \mathrm{p}$. 37]. There is a second Radau rule symmetric to this one with respect to the origin but it suffices to discuss the first one. The error in the numerical integration of $T_{m}(x)$ and $U_{m}(x)$ by such a rule is zero for $m \leqq 2 n$. Hence we are concerned with $E_{n+1}^{R}\left(T_{2 n+k}\right)$ and $E_{n+1}^{R}\left(U_{2 n+k}\right), k \geqq 1$.

Now, by the Darboux formula for $P_{n}^{(\alpha, \beta)}(\cos \theta)$ [2, p. 194],

$$
P_{n}^{(\alpha, \beta)}(\cos \theta)=n^{-1 / 2} k(\theta) \cos (N \theta+\phi)+O\left(n^{-3 / 2}\right)
$$

where $k(\theta)=\pi^{-1 / 2}(\sin \theta / 2)^{-\alpha-1 / 2}(\cos \theta / 2)^{-\beta-1 / 2}, N=n+(\alpha+\beta+1) / 2$,

$$
\phi=-(\alpha+1 / 2) \pi / 2, \quad 0<\theta<\pi,
$$

we see that the asymptotic behavior of $P_{n}^{(1,0)}(x)$ is given by

$$
P_{n}^{(1,0)}(\cos \theta)=-\left(\frac{2}{n \pi \sin \theta}\right)^{1 / 2} \frac{\sin [(n+1) \theta-\pi / 4]}{\sin \theta / 2}+O\left(n^{-3 / 2}\right) \text {. }
$$

Thus

$$
\left[P_{n}^{(1,0)}(\cos \theta)\right]^{2}(1-\cos \theta)=\frac{2[1-\sin (2 n+2) \theta]}{n \pi \sin \theta}+O\left(n^{-2}\right)
$$




\section{TABLE II}

Asymptotic Error as $n \rightarrow \infty$ in the Integration of $T_{m}$ and $U_{m}$ by Gauss, Lobatto and Radau Rules

\begin{tabular}{|c|c|c|c|c|c|c|}
\hline & Gauss & $n$-Points & Lobatto & $(n+1)$-Points & Radau & $(n+1)$-Points \\
\hline$k$ & $T_{2 n+2 k}(x)$ & $U_{2 n+2 k}(x)$ & $T_{2 n+2 k}(x)$ & $U_{2 n+2 k}(x)$ & $T_{2 n+1+k}(x)$ & $U_{2 n+1+k}(x)$ \\
\hline 0 & $\frac{\pi}{2}+o\left(\frac{1}{n}\right)$ & $\pi+o\left(\frac{1}{n}\right)$ & $-\frac{\pi}{2}+o\left(\frac{1}{n}\right)$ & $-\pi+o\left(\frac{1}{n}\right)$ & $-\frac{\pi}{2}+O\left(\frac{1}{n}\right)$ & $-\pi+o\left(\frac{1}{n}\right)$ \\
\hline 1 & $\frac{\pi}{2}+o\left(\frac{1}{n}\right)$ & $O\left(\frac{1}{n}\right)$ & $\frac{\pi}{2}+o\left(\frac{1}{n}\right)$ & & $O\left(\frac{1}{n}\right)$ & \\
\hline 2 & & $O\left(\frac{1}{n}\right)$ & $O\left(\frac{1}{n}\right)$ & $O\left(\frac{1}{n}\right)$ & $\frac{\pi}{2}+O\left(\frac{1}{n}\right)$ & \\
\hline 3 & & $O\left(\frac{1}{n}\right)$ & & $O\left(\frac{1}{n}\right)$ & $O(1$ & $o\left(\frac{1}{n}\right)$ \\
\hline
\end{tabular}

and by analogy to (4) and (5) we have

$$
\begin{aligned}
T_{2 n+k}(\cos \theta)= & \left\{\frac{2[1-\sin (2 n+2) \theta]}{n \pi \sin \theta}+O\left(n^{-2}\right)\right\} \sum_{j=0}^{k-1} d_{i}^{(k)} \cos \theta j \\
& +Q_{2 n}(\cos \theta), \\
U_{2 n+k}(\cos \theta)= & \left\{\frac{2[1-\sin (2 n+2) \theta]}{n \pi \sin \theta}+O\left(n^{-2}\right)\right\} \sum_{j=0}^{k-1} e_{j}^{(k)} \cos \theta j \\
& +R_{2 n}(\cos \theta) .
\end{aligned}
$$

Proceeding along lines similar to that in the previous section, we get the following relations:

$$
\begin{array}{ll}
d_{i}^{(k)}=-\frac{1}{2} n \pi\left[\delta_{(k-1) i}-\delta_{(k-3) i}\right]+O(1), & j=0, \cdots, k-1, k \geqq 2, \\
d_{0}^{(1)}=-n \pi / 4+O(1), & j=0, \cdots, k-1, k \geqq 2, \\
e_{i}^{(k)}=-n \pi \delta_{(k-1) i}+O(1), & \\
e_{0}^{(1)}=-n \pi / 2+O(1) . &
\end{array}
$$

Inserting these into (13) and (14), we get the following asymptotic expressions:

$$
\begin{aligned}
T_{2 n+k}(\cos \theta)= & \frac{\sin (2 n+2) \theta-1}{\sin \theta}[\cos (k-1) \theta-\cos (k-3 \theta)] \\
& +O\left(n^{-1}\right)+Q_{2 n}(\cos \theta), \quad k \geqq 3, \\
T_{2 n+2}(\cos \theta)= & \frac{\sin (2 n+2) \theta-1}{\sin \theta} \cos \theta+O\left(n^{-1}\right)+Q_{2 n}(\cos \theta),
\end{aligned}
$$




$$
\begin{aligned}
T_{2 n+1}(\cos \theta)= & \frac{\sin (2 n+2) \theta-1}{2 \sin \theta}+O\left(n^{-1}\right)+Q_{2 n}(\cos \theta), \\
U_{2 n+k}(\cos \theta)= & \frac{2 k \sin (2 n+2) \theta-1}{2 \sin \theta} \cos (k-1) \theta+O\left(n^{-1}\right) \\
& +R_{2 n}(\cos \theta), \quad k \geqq 2, \\
U_{2 n+1}(\cos \theta)= & \frac{\sin (2 n+2) \theta-1}{\sin \theta}+O\left(n^{-1}\right)+R_{2 n}(\cos \theta) .
\end{aligned}
$$

From these, we derive our final results:

$$
\begin{aligned}
& E_{n+1}^{R}\left(T_{2 n+k}\right)=O\left(n^{-1}\right), \quad k=2, \quad k \geqq 4, \\
& E_{n+1}^{R}\left(T_{2 n+3}\right)=\pi / 2+O\left(n^{-1}\right), \\
& E_{n+1}^{R}\left(T_{2 n+1}\right)=-\pi / 2+O\left(n^{-1}\right), \\
& E_{n+1}^{R}\left(U_{2 n+k}\right)=O\left(n^{-1}\right), \quad k \geqq 2, \\
& E_{n+1}^{R}\left(U_{2 n+1}\right)=-\pi+O\left(n^{-1}\right) .
\end{aligned}
$$

In Table II, we summarize the asymptotic behavior of the errors as $n \rightarrow \infty$ in the integration of $T_{m}(x)$ and $U_{m}(x)$ by Gauss, Lobatto and Radau integration rules.

Department of Computer Science

University of Edinburgh

Edinburgh, Scotland, U.K.

Department of Applied Mathematics

Weizmann Institute of Science

Rehovot, Israel

1. P. J. Davis \& P. Rabinowitz, Numerical Integration, Blaisdell, Waltham, Mass., 1967. MR 35 \#2482.

2. G. Szegö, Orthogonal Polynomials, 2nd rev. ed., Amer. Math. Soc. Colloq. Publ., vol. 23, Amer. Math. Soc., Providence, R. I., 1959. MR 21 \#5029. 